\title{
Coherent control of surface plasmon polariton mediated optical transmission
}

\author{
L Le Guyader ${ }^{1}$, A Kirilyuk ${ }^{1}$, Th Rasing ${ }^{1}$, G A Wurtz ${ }^{2}$, A V Zayats ${ }^{2}$, \\ P F A Alkemade ${ }^{3}$ and I I Smolyaninov ${ }^{4}$ \\ ${ }^{1}$ IMM, Radboud University Nijmegen, Toernooiveld 1, 6525ED Nijmegen, The Netherlands \\ ${ }^{2}$ Centre for Nanostructured Media, IRCEP, The Queen's University of Belfast, Belfast BT7 1NN, UK \\ ${ }^{3}$ Kavli Institute of Nanoscience, Delft University of Technology, Lorentzweg 1, 2628 CJ Delft, \\ The Netherlands \\ ${ }^{4}$ Department of Electrical and Computer Engineering, University of Maryland, College Park, MD, USA \\ E-mail: 1.leguyader@science.ru.nl
}

Received 10 June 2008, in final form 31 July 2008

Published 11 September 2008

Online at stacks.iop.org/JPhysD/41/195102

\begin{abstract}
We demonstrate the coherent control of the surface plasmon polariton mediated optical transmission through arrays of nano-holes in a gold film on a garnet substrate by a standing surface acoustic wave (SAW) induced by a sequence of ultrashort laser pulses. The transmission of the pump pulse through the holes results in a periodic pattern of shock-waves leading, via interference, to a standing SAW. The resulting modulation frequency is determined by the periodicity of the arrays. The polarization of the transmitted light is modulated as well via a strain-induced linear dichroism.
\end{abstract}

(Some figures in this article are in colour only in the electronic version)

Surface plasmon polariton (SPP) subwavelength optics is considered to be very promising for the realization of miniaturized photonic circuits [1]. SPPs are electromagnetic excitations confined at the interface between a metal and a dielectric [2]. The ability to create well-defined nanoscale structures in metal films allows engineering of SPP states and has already led to the discovery of several phenomena $[3,4]$, such as the enhancement of fluorescence of single molecules [5], second harmonic generation [6] and the extraordinary optical transmission (EOT) [7,8] of light through subwavelength apertures. However, all these examples rely on static control of the SPP properties, while to realize functional plasmonic circuits, dynamic control is required. For example, in the case of the EOT, one would like to have external control of the transmission [9-11].

Ultrafast coherent control of the SPP can be achieved by shaping the laser pulse which is used to induce them [1214]. However, this supposes, in the case of EOT, direct modulation of the transmitted light, losing the information transported. Another approach would be to coherently modify parametrically the SPP by an external stimulus. For example, modifying the dielectric function of the metal $\epsilon_{\mathrm{m}}$ or the dielectric $\epsilon_{\mathrm{d}}$ at the interface will lead to a modification of the transmission spectrum. The switching of a liquid crystal filled array of holes has been shown to lead to a change in transmission of the structure $[9,15]$. However, the speed of this process, which is on the order of a few microseconds, is too slow to compete with the current technologies for information processing. The thermally induced change between the two metallic phases of a gallium film by a control light beam results in a significant change in the $\epsilon_{\mathrm{m}}$ and subsequently a change in the transmission [16], which lasts for about $50 \mathrm{~ns}$. Even faster approaches involve strong ultrashort laser pulses, which, when absorbed by the metal, change the electron distribution around the Fermi level. This leads to a transient change in the dielectric function [17] and as a result, to a transient change in the transmission on a timescale of about a few picoseconds in metals [18] and semiconductors [19] or adjacent dielectric $[10,11]$. However, in all these examples, once the change is induced, one has to wait for the natural relaxation of the process involved before taking the next step in control, which thus lacks real dynamical control of the SPP.

A solution to this problem may be to use standing surface acoustic waves (SAWs). These waves can be generated by a laser pulse in structures similar to those showing EOT [20-22]. SAWs are known to change the dielectric function 
of the material in which they propagate via the photoelastic effect [23] and coherent control of acoustical phonons has been demonstrated [24].

In this work we demonstrate that coherent control of the SPP-mediated optical transmission by a standing SAW can be obtained on subnanosecond timescales, using a proper pulse sequence. The standing SAW is optically generated by an ultrashort laser pulse absorbed in a dielectric garnet through a metallic array of subwavelength nano-holes. The frequency of this standing SAW is given by the periodicity between the holes of the array and is of the order of several gigahertz. This standing SAW then modulates the SPP-mediated optical transmission. The polarization of the transmitted light is also modulated on the same timescale via an induced linear dichroism.

The samples were made of $3.5 \mu \mathrm{m}$ thick single-crystalline iron garnet (IG) films of composition $\mathrm{Lu}_{2.4} \mathrm{Bi}_{0.6} \mathrm{Fe}_{4.8} \mathrm{Ga}_{0.2} \mathrm{O}_{12}$ which is elastically isotropic [25] and grown by isothermal liquid phase epitaxy on a $\left(\begin{array}{llll}0 & 0\end{array}\right)$ oriented gadolinium gallium garnet (GGG) substrate. On top of the IG, a $80 \mathrm{~nm}$ thick polycrystalline gold film was deposited, in which various structures were milled with a focused ion beam (FIB). $25 \times$ $25 \mu \mathrm{m}^{2}$ arrays of nano-holes were fabricated with a periodicity ranging from 200 to $600 \mathrm{~nm}$ with steps of $50 \mathrm{~nm}$ and with hole diameters of 60,100 and $150 \mathrm{~nm}$.

Time resolved measurements on all these structures were performed with an optical pump-probe setup with 100 fs laser pulses at $808 \mathrm{~nm}$ wavelength, amplified to $1 \mathrm{~mJ}$ of energy per pulse, with a repetition rate of $1 \mathrm{kHz}$. Those pulses were split into a low and high intensity part by a glass wedge. The higher intensity pulses were used as a pump after their frequency was doubled by a BBO crystal to $404 \mathrm{~nm}$ at which wavelength the IG is strongly absorbing. The fluence of each individual pump pulse was about $3 \mathrm{~mJ} \mathrm{~cm}^{-2}$ over all the $25 \times 25 \mu \mathrm{m}^{2}$ arrays, while the probe pulse fluence was about 100 times weaker. The probe beam remained at $808 \mathrm{~nm}$ where the IG is transparent. The delay between the pump and the probe could be adjusted up to $3 \mathrm{~ns}$ with a resolution better than $10 \mathrm{fs}$ by a delay line in the pump beam path. A synchronized chopper was used for the pump modulation which blocked every second pump pulse, resulting in a pump frequency of $500 \mathrm{~Hz}$. After passing through the sample, the probe pulse was split into two orthogonal linearly polarized beams, and their intensity was detected by two independent identical diodes. The sum and the difference of the signal of these two diodes, detected with two lock-in amplifiers synchronized to the pump pulse frequency of $500 \mathrm{~Hz}$, permit simultaneous determination of the relative change in the probe transmitted intensity $\Delta I$ (normalized by transmitted intensity in the absence of pump pulses) and the polarization rotation of the transmitted probe light $\Delta \rho$, respectively.

Figure 1 shows typical time resolved measurements of the change in intensity $\Delta I$ and the polarization rotation $\Delta \rho$ of the probe pulse transmitted through an array of holes, in the configuration shown in figure 2. One can see that after the pump arrived on the sample, oscillations appear in both the change in intensity $\Delta I$ and the polarization rotation $\Delta \rho$. This can be explained as follows. The ultrashort laser pump

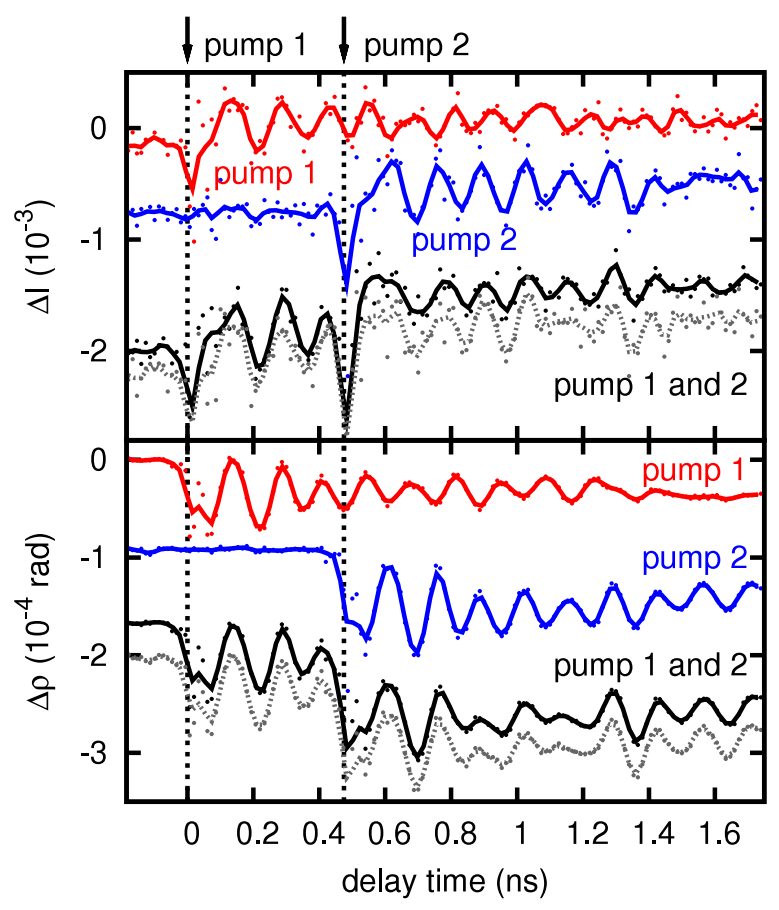

Figure 1. Time resolved measurements of the change of intensity $\Delta I$ and polarization rotation $\Delta \rho$ of the transmitted probe light through an array of holes having a periodicity of $300 \mathrm{~nm}$ and a hole diameter of $150 \mathrm{~nm}$. The dashed curve corresponds to the sum of the curve labelled 'pump 1' and the curve labelled 'pump 2' while the continuous line labeled 'pump 1 and 2' corresponds to the measurement with both pumps.

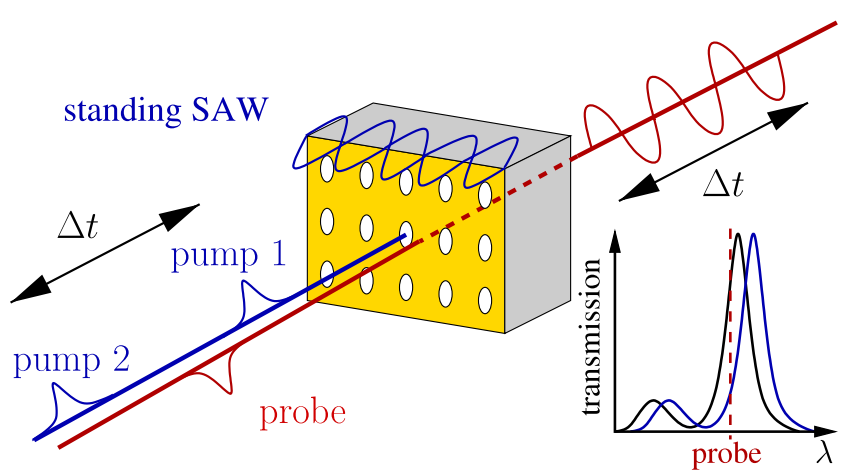

Figure 2. Experimental scheme of the coherent control of the modulation of the transmitted probe light through an array of nano-holes by the standing SAW with a sequence of pump pulses. The inset shows the modification of the SPP-mediated transmission by the standing SAWs induced by the pump pulses.

pulse absorbed by the substrate creates shock-waves from each individual hole, which then interfere to create a standing SAW. The frequency $f$ of this standing SAW is given by $f=v_{\mathrm{R}} / p$, where $p$ is the periodicity between the holes and $v_{\mathrm{R}}$ the surface sound velocity. Measurements through other arrays show a constant $v_{\mathrm{R}}$ of $2.0 \mathrm{~km} \mathrm{~s}^{-1}$, which is in reasonable agreement with the velocity of $2.4 \mathrm{~km} \mathrm{~s}^{-1}$ calculated for the IG of this composition. These waves then change the SPP dispersion by modulating the dielectric constant of the garnet via the photoelastic effect [22]. This results in the observed modulation of the transmitted intensity, while an induced linear dichroism modulates the polarization of the transmitted light. 


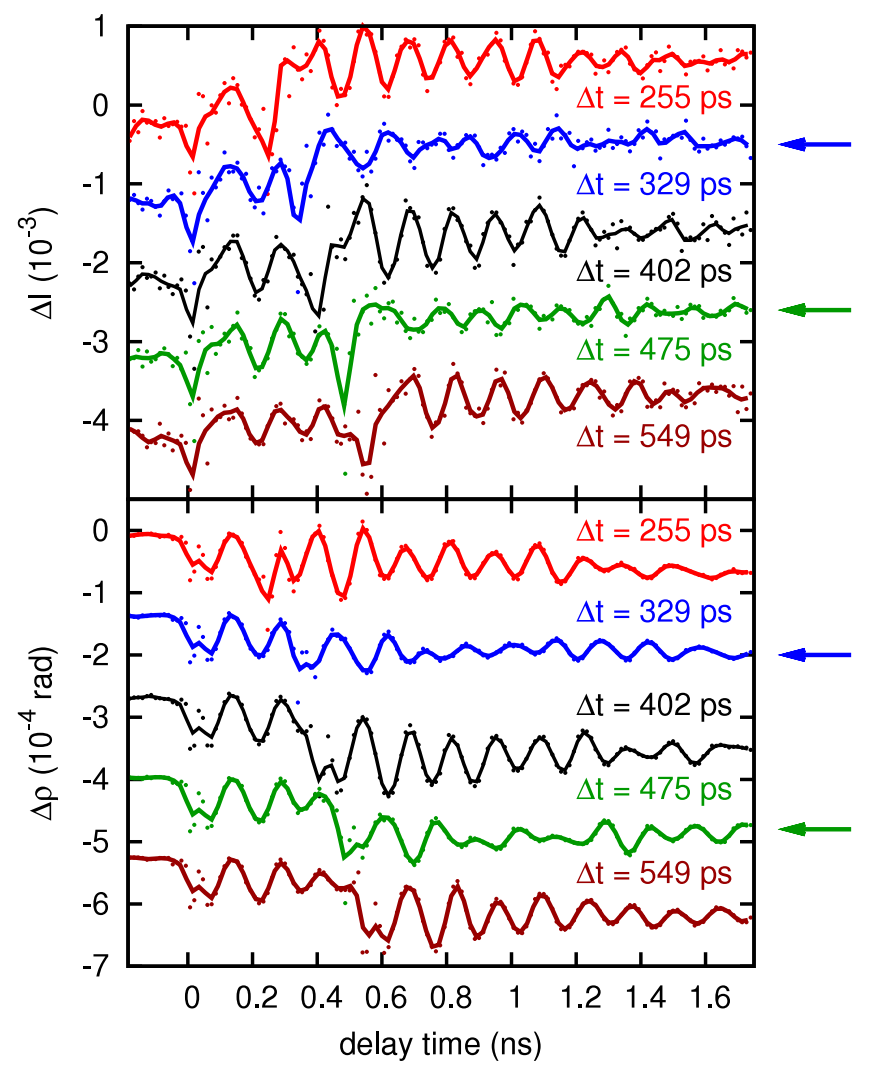

Figure 3. Time resolved measurement of the change in intensity $\Delta I$ and polarization rotation $\Delta \rho$ of the transmitted probe light through an array of holes having a periodicity of $300 \mathrm{~nm}$ and a hole diameter of $150 \mathrm{~nm}$ for different delays $\Delta t$ of the second pump given by the label of the curve. The arrows indicate cases of near cancellation.

This pump-induced SPP dispersion change is the most effective mechanism which contributes to the periodic modulation of the transmitted light intensity through this subwavelength nano-holes structure.

Figure 1 also displays what happens if one uses a second pump to excite a second standing SAW with a delay corresponding to the creation of opposite phase oscillations with respect to the previous pump. The amplitude of the oscillations in the change in intensity $\Delta I$ and the polarization rotation $\Delta \rho$ is reduced compared with the case of only one pump. In addition, the result of using two pump pulses corresponds exactly to the superposition of the effect of two pump pulses independently, as shown by the dashed curve in figure 1, which means that coherent control is possible.

By changing the delay between the two pump pulses, we observe that the amplitude of the oscillations in the change in intensity $\Delta I$ and the polarization rotation $\Delta \rho$ passes alternately through a minimum and a maximum, as shown in figure 3 . The periodicity between successive minima and maxima is found to be $73 \mathrm{ps}$ in this case. This is in very good agreement with the half period $T_{1 / 2}=75 \mathrm{ps}$ of the acoustic oscillations in this structure, given by $T_{1 / 2}=p / 2 v_{\mathrm{R}}$, where $p=300 \mathrm{~nm}$ is the periodicity of the array and $v_{\mathrm{R}}=2.0 \mathrm{~km} \mathrm{~s}^{-1}$ is the velocity of the SAWs. This shows that two pump pulses can be used to start and stop the oscillatory transmission of the light through the array of holes in a controlled manner.

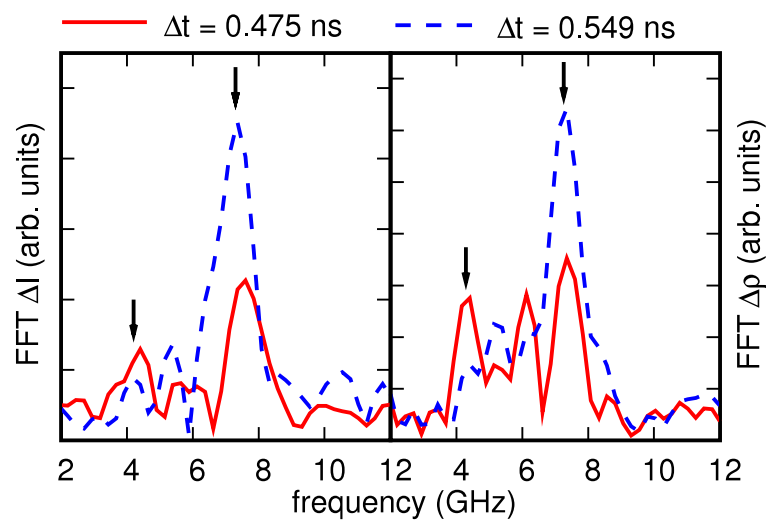

Figure 4. Fourier transform of the time dependent change in intensity $\Delta I$ and polarization rotation $\Delta \rho$ of the transmitted probe light through an array of holes having a periodicity of $300 \mathrm{~nm}$ and a hole diameter of $150 \mathrm{~nm}$ for two different delays $\Delta t$ of the second pump pulse. Arrows indicate the 2 modes discussed in the text.

However, it is also clear that some oscillations remain in the case of a minimum, which means that the quenching is not perfect. This is partly due to the fact that the standing SAWs are damped within the time frame during which coherent control is attempted. This means that the power of the second pump pulse should be tuned for each delay between the two pump pulses to get an exact cancellation. This fast decay of the standing SAW is probably due to the thickness of the gold, which loads the surface of the IG and forces the standing SAW to radiate energy into the bulk $[21,26]$. In addition, several frequencies are present, as shown in figure 4. One can see that the power $P$ in the main peak corresponding to the standing SAW depends on the time between the pumps, and that it can be reduced $\left(P_{\mathrm{r}}\right)$ or amplified $\left(P_{\mathrm{a}}\right)$, with a contrast of $\left(P_{\mathrm{a}}-P_{\mathrm{r}}\right) /\left(P_{\mathrm{a}}+P_{\mathrm{r}}\right)=30 \%$. It also shows that other modes are present in the spectra, in particular one at $4.4 \mathrm{GHz}$, which is amplified when the main mode is attenuated, as indicated by arrows in figure 4 . This mode dephasing is also partly responsible for the non ideal cancellation of the observed oscillations. These other frequencies are partly due to the non ideal quality of the structures, in particular the dispersion of the periodicity and the hole shape within an array. However, it is not a fundamental limit and it can easily be solved with improved technology of samples fabrication. These acoustic frequencies also arise from the different possible vectors of the reciprocal lattice of a square array of holes. This can be overcome by using different structure geometries, such as slits or concentric rings. Moreover, the damping of the standing SAW can be reduced by using a thinner gold film or a lighter metal. It should thus be possible to excite a single undamped mode for which the coherent control would be very precise. It would also be interesting to evaluate the outcome of similar experiments with three or four pump pulses to see whether better control can also be achieved for a multi-mode system.

In conclusion, we have shown that the SPP-mediated optical transmission through arrays of nano-holes can be modulated at several gigahertz and that this modulation can be coherently controlled by a sequence of ultrafast laser pulses, via the coherent control of the standing SAWs. These standing 
SAWs also induce a linear dichroism which modulates the polarization of the transmitted light and can be coherently controlled as well. This work is thus a step towards the development of performant devices, in particular for the ultrafast processing of optical information.

\section{Acknowledgments}

We thank the EU projects DYNAMICS, the Dutch organisation for Fundamental Research on Matter (FOM) and the NanoNed consortium for financial support. This work was in part supported by the EPSRC (UK).

\section{References}

[1] Barnes W L, Dereux A and Ebbesen T W 2003 Nature 424824

[2] Raether H 1988 Surface Plasmons on Smooth and Rough Surfaces and on Gratings (Berlin: Springer)

[3] Zayats A V, Smolyaninov I I and Maradudin A A 2005 Phys. Rep. 408131

[4] Genet C and Ebbesen T W 2007 Nature 44539

[5] Rigneault H, Capoulade J, Dintinger J, Wenger J, Bonod N, Popov E, Ebbesen T W and Lenne P-F 2005 Phys. Rev. Lett. 95117401

[6] van Nieuwstadt J A H, Sandtke M, Harmsen R H, Segerink F B, Prangsma J C, Enoch S and Kuipers L 2006 Phys. Rev. Lett. 97146102

[7] Ebbesen T W, Lezec H J, Ghaemi H, Thio T and Wolff P A 1998 Nature 391667

[8] Ghaemi H F, Thio T, Grupp D E, Ebbesen T W and Lezec H J 1998 Phys. Rev. B 586779
[9] Dickson W, Wurtz G, Evans P, Pollard R and Zayats A 2008 Nano Lett. 8281

[10] Wurtz G A, Pollard R and Zayats A V 2006 Phys. Rev. Lett. 97057402

[11] Smolyaninov I I, Zayats A V, Stanishevsky A and Davis C C 2002 Phys. Rev. B 66205414

[12] Stockman M I, Faleev S V and Bergman D J 2002 Phys. Rev. Lett. 88067402

[13] Sukharev M and Seideman T 2007 J. Phys. B: At. Mol. Opt. Phys. 40 S283

[14] Aeschlimann M, Bauer M, Bayer D, Brixner T, de Abajo F J G, Pfeiffer W, Rohmer M, Spindler C and Steeb F 2007 Nature 446301

[15] Kim T J, Thio T, Ebbesen T W, Grupp D E and Lezec H J 1999 Opt. Lett. 24256

[16] Krasavin A V, MacDonald K F, Zheludev N I and Zayats A V 2004 Appl. Phys. Lett. 853369

[17] Hohlfeld J, Wellershoff S S, Gudde J, Conrad U, Jahnke V and Matthias E 2000 Chem. Phys. 251237

[18] Halt V, Benabbas A, Guidoni L and Bigot J Y 2005 Phys. Status Solidi b 2421872

[19] Hendry E, Lockyear M J, Rivas J G, Kuipers L and Bonn M 2007 Phys. Rev. B 75235305

[20] Bennis A, Lomonosov A M, Shen Z H and Hess P 2006 Appl. Phys. Lett. 88101915

[21] Bonello B, Ajinou A, Richard V, Djemia P and Chrif S M 2001 J. Acoust. Soc. Am. 1101943

[22] Gerard D, Laude V, Sadani B, Khelif A, Labeke D V and Guizal B 2007 Phys. Rev. B 76235427

[23] Mueller H 1935 Phys. Rev. 47947

[24] Özgür U, Lee C-W and Everitt H O 2001 Phys. Rev. Lett. 865604

[25] Slack G A and Oliver D W 1971 Phys. Rev. B 4592

[26] Lin H N, Maris H J, Freund L B, Lee K Y, Luhn H and Kern D P 1993 J. Appl. Phys. 7337 